\title{
A Cyber-Physical System to Improve the Management of a Large Suite of Operating Rooms
}

\author{
GUILLAUME JOERGER, Center for Computational Surgery, Houston Methodist Hospital - \\ Houston, USA \\ JULIETTE RAMBOURG, ENAC French University of Civil Aviation - Toulouse, France, \& Center for \\ Computational Surgery Houston Methodist Hospital - Houston, USA \\ HELENE GASPARD-BOULINC and STEPHANE CONVERSY, ENAC French University of Civil \\ Aviation - Toulouse, France \\ BARBARA L. BASS and BRIAN J. DUNKIN, Department of Surgery, Houston Methodist Hospital - \\ Houston USA \\ MARC GARBEY, Center for Computational Surgery, Houston Methodist Hospital - Houston USA
}

\begin{abstract}
Cyber-physical systems have been deployed with considerable success in many industries. However, the implementation of cyber-physical systems in hospitals has been limited. By nature, in clinical operations, patient safety and consideration for health outcomes are of the utmost importance, thus possibly slowing the implementation of innovative solutions with limited history. Revenues from operating room (OR) time and surgery account for about 50\% of the income of major hospitals (Erdogan et al. 2011; Cuschieri 2006), but the efficiency of OR utilization is often reported to be relatively low. Therefore, improving OR management with a cyber-physical system should be a priority. In this article, we will report on our experience implementing a cyber-physical system at Houston Methodist Hospital and discuss some of the difficulties and potential drivers for success. Our pilot study was done in the context of the management of a large suite of ORs. It uses the agile codevelopment of a cyber-physical system through an intense collaboration of clinicians and computational scientists. While technology remains the foundation of a cyber-physical system, this experience reinforced that the human factor is an important driving force behind the design that promotes user acceptance.
\end{abstract}

CCS Concepts: • Human-centered computing $\rightarrow$ Human computer interaction (HCI); • Applied computing $\rightarrow$ Health care information systems; • Computer systems organization $\rightarrow$ Embedded and cyber-physical systems; • Hardware $\rightarrow$ Signal processing systems; Sensors and actuators; • Computing methodologies $\rightarrow$ Modeling and simulation;

Additional Key Words and Phrases: Cyber-physical system, hospital operating room, smart sensors, human computer interface, agile development, agent-based model

Preparation of this article was supported by the National Science Foundation under the I/UCRC for Cyber-Physical Systems for the Hospital Operating Room Grant No. 1657550.

Authors' addresses: G. Joerger and M. Garbey, PhD, Center for Computational Surgery, Houston Methodist Hospital Research Institute, 6670 Bertner Av., Houston, TX 77030, USA and LaSIE, UMR CNRS 7356, Universite de La Rochelle, France; emails: \{gjoerger, mgarbey2\}@houstonmethodist.org; J. Rambourg, ENAC French University of Civil Aviation Toulouse, France and Center for Computational Surgery, Houston Methodist Hospital Research Institute, 6670 Bertner Av., Houston, TX 77030, USA; email: jrambourg@houstonmethodist.org; H. Gaspard-Boulinc and S. Conversy, ENAC French University of Civil Aviation, 7 avenue Edouard Belin, 31400 Toulouse, France; emails: \{helene.gaspard-boulinc, stephane.conversy\}@enac.fr; B. L. Bass and B. J. Dunkin, MD, Department of Surgery, Houston Methodist Hospital, 6550 Fannin St., Houston, TX 77030, USA; emails: \{BBass, BJDunkin\}@houstonmethodist.org.

Permission to make digital or hard copies of all or part of this work for personal or classroom use is granted without fee provided that copies are not made or distributed for profit or commercial advantage and that copies bear this notice and the full citation on the first page. Copyrights for components of this work owned by others than ACM must be honored. Abstracting with credit is permitted. To copy otherwise, or republish, to post on servers or to redistribute to lists, requires prior specific permission and/or a fee. Request permissions from permissions@acm.org.

(C) 2018 ACM 2378-962X/2018/07-ART34 \$15.00

https://doi.org/10.1145/3140234

ACM Transactions on Cyber-Physical Systems, Vol. 2, No. 4, Article 34. Publication date: July 2018. 


\section{ACM Reference format:}

Guillaume Joerger, Juliette Rambourg, Helene Gaspard-Boulinc, Stephane Conversy, Barbara L. Bass, Brian J. Dunkin, and Marc Garbey. 2018. A Cyber-Physical System to Improve the Management of a Large Suite of Operating Rooms. ACM Trans. Cyber-Phys. Syst. 2, 4, Article 34 (July 2018), 24 pages.

https://doi.org/10.1145/3140234

\section{INTRODUCTION}

The operating room $(\mathrm{OR})$ is by far the most complex and expensive environment within any hospital. To start a case, it is necessary to ensure that all staff including the surgeon, anesthesiologist, scrub tech, and technician are ready and context aware and that all equipment is available and functional. However, a surgery is highly patient specific and carries many sources of uncertainty, thus making a prediction of surgery time very challenging. Our study focused on optimizing the management of a large suite of ORs at Houston Methodist Hospital, which includes 23 ORs. Key resources, such as personnel, for example, anesthesiologists and technicians, as well as specialized pieces of equipment, such as 3D ultrasound and laparoscopy tools, are dynamically allocated within the OR suite. Optimizing OR management is by nature a real-time process that cannot rely exclusively on scheduling done in advance. Organizing the surgery flow is a very difficult problem within a complex system that has many unpredictable parts. A significant number of surgical procedures are either delayed or canceled because a preceding surgery lasted much longer than expected and/or did not start on time, therefore delaying or forcing the cancellation of other scheduled procedures (Gillen et al. 2009; Argo et al. 2009; Sanjay et al. 2007). It is estimated that OR time cost is about $\$ 100$ per minute (Macario et al. 2001, 2010), so OR inefficiency has a high impact on hospital revenue and eventually health care cost. Patient outcomes might also benefit from an efficient system that improves the organization so that time spent under anesthesia is minimized. In addition, increased efficiency may decrease stress and frustration arising from unexpected wait times for patients and OR staff. Medical Cyber-Physical Systems (MCPSs) are life-critical, context-aware, and/or networked systems of medical devices (Lee 2015). Developing MCPSs for the surgical suite management is feasible and the next step forward in health care innovation leading to increased efficiency, cost savings, improved patient outcomes, and better health care.

To our surprise, there are few successful medical cyber-physical systems reported in the literature and even fewer used daily in the clinic. One may argue that safety is a big risk that explains why MCPSs are difficult to implement. However, equally critical safety systems such as the power grid or air civil transportation have existing cyber-infrastructure that is used daily, has proven reliable and effective, and is an integral part of everyday life. It is worth pointing out the parallels between the past deployment of the Electronic Medical Record (EMR) in hospitals and the trajectory of MCPS adoption. Widespread adoption of EMRs in hospital systems took a long time despite the fact that health insurer and government policies were making it progressively mandatory.

Furthermore, a system like Epic, which is taking the dominant position in the EMR market with an $80 \%$ market share (HIMSS analytics Database 2015), is still built on a client-server architecture and a programming language MUMPS developed in the 1960s when memory was a limitation and programmers were using lots of now-obsolete abbreviations (Koppel et al. 2014). As a result, this software is not ready to be used with other languages, especially cloud-ready ones, thus slowing down the system itself-its responsiveness, improvements, and updates-and restrains innovation in the health care industry globally (http://www.carecloud.com/continuum/ehr-languageseries-mumps/). 
This report details our experience at Houston Methodist Hospital with a pilot study that focused exclusively on improving the management of a large suite containing 23 ORs. Our MCPS is the combination of two projects: the smartOR and BoardProbe. The smartOR is a distributed wireless network of sensors aimed at automatically detecting key steps of OR activity. Its goal is to reduce manual record entry by nurses and anesthesiologists and provide near real-time information on the progress of surgeries to OR management and staff through nonintrusive sensors and computation. BoardProbe is a technology probe (Hutchinson et al. 2003) consisting of an application on a large multitouch screen, which assists the surgical suite staff's collaborative activity and provides the information gathered by the smartOR to the staff in a real time. It has three underlying principles: social science, engineering, and design. By connecting BoardProbe to the smartOR, we bring context awareness to the OR as well as the perioperative area. We support individual and collaborative staff activities while maintaining a patient-centric focus.

In this study, we focus on four different aspects of OR management through cyber-physical systems. First, by studying a surgical suite facility in our institution and the surgical suite staff, we deliver a better description of the activity and the needs of the staff to perform their task. Second, by bringing cyber-physical technologies to the ORs and other parts of the surgical suite, we help to develop computerization of a critical environment under operating conditions and deliver an unbiased and near-real-time status of the ORs within the surgical suite that would otherwise require manual input. Third, we present the results of clinical tests of the smartOR hardware and software that have been performed over the course of the last year and provide some analytical tools. Fourth, we discuss a method to custom design and implement MCPSs in other hospital systems. The emphasis of this article is not just on the technology, despite the fact that it is rather challenging to deploy new technologies in the context of very strict requirements imposed by health care organizations regulations, but rather on the unique codevelopment of the cyber-physical system through intense daily collaboration between clinicians and computational scientists in order to improve the system and gain user acceptance. Our hope is that our contribution can offer a pathway to a better design and promote the best cyber-physical system practices already available in other industries like energy (Karnouskos 2011) and civil aviation (Sampigethaya and Poovendran 2013).

To summarize, the targeted problem is the improvement of surgical suite awareness and workflow analysis by developing a system that acquires and transmits surgical flow data to the surgical staff. The contributions described in this article consist of an ethnographic study of the surgical suite staff within a large surgical suite, the design and implementation of noninvasive sensors for the OR (inputs), a network of perioperative area interfaces to transmit the data (inputs and outputs), and a mathematical model that detects possible efficiency improvements in the workflow and supports staff decisions.

\section{RELATED WORK}

Designing an effective MCPS for the management of the hospital OR is a highly multidisciplinary activity. This section briefly summarizes the literature describing some key elements, though a thorough review is beyond the scope of this article.

\subsection{Cyber-Physical Systems for Activity Management}

Even though MCPS research often targets study at the device level, several interesting research projects emerged using CPS to improve the management of large-scale critical activities. The National Institute of Standards and Technology (NIST) highlights this need at the scale of the city on its website (http://www.nist.gov/cps/), suggesting that it will improve our performances in various fields such as health care, emergency response, traffic flow management, energy, and others. 
For example, the smart firefighters' activity reported at a NIST workshop summary (Hamins et al. 2014) revealed that CPS, by tracking data (thermal, smoke, etc., within a fire structure) is able to improve firefighters' awareness. In the energy field, smart grid projects are very promising (Karnouskos 2011). Civil aviation is implementing high-level flight management systems to optimize flight routes including many constraints (fuel, noise, and weather) and improve automation and decision support in the cockpit (Sampigethaya and Poovendran 2013).

\subsection{Medical Cyber-Physical Systems Review}

Lee et al. (2012) pinpointed six significant challenges for MCPS: high assurance software, interoperability, context awareness, autonomy, security and privacy, and certifiability. The authors mention the importance of following a coordinated model that ensures these six points are considered for any MCPS. Li et al. (2015) emphasize the same factors and present some MCPS success stories. MCPSs are limited by the fact that they typically serve to solely interconnect medical devices and, in general, are unable to detect human errors. Further, the authors identified two specific challenges for MCPSs:

1. Understanding staff activity and how the staff interacts with MCPSs (context awareness)

2. Uncertainty of the acquired data

We face the same challenges with the system we present in this article and thus accentuate our description of the staff activity analysis and our integrative collaboration with them. However, our approach is unique in that we employ a cluster of sensors in conjunction with nonmedical devices to capture OR context.

\subsection{Examples of Computer Systems for OR Surgical Procedure Management}

Bardram et al. (2008) report the successful Context-Aware Patient Safety and Information System (CAPSIS) experiment, tested during a 1-day experiment at a hospital with a real surgery team and patient actors. Despite the success of the experiment evidenced by the statement that "the clinicians concluded that the system would be very useful in ensuring patient safety and that it was very easy to use," to our knowledge CAPSIS is not yet routinely used in the clinic.

Bardram (2009), Bardram et al. (2005), Scupelli et al. (2010), and Tang et al. (2009) also focused on collaboration within the surgical suite. For Bardram (2009), computerization in hospitals is a problem since the systems are "typically single-user oriented," whereas teamwork is omnipresent. These studies describe activities within the surgical suite of other hospitals, sometimes around the same kind of whiteboards studied in our work. While the authors provide recommendations for designing collaborative tools for the surgical suite, these studies did not develop collaborative tools themselves or test their design. This aspect is also missing in Computer-Supported Collaborative Work (CSCW) for the hospitals (Fitzpatrick et al. 2013). Here the authors state that, for the past 20 years, the commercially available electronic whiteboards have not been adequate. According to the study, the electronic whiteboard lacks usability and the display and hardware are of poor quality.

Bossen et al. (2015) recommend deploying pliant systems by "moving from design-for-use to design-for-future-use." The design choice of the authors to improve collaboration in the "Care Hotel," a "municipal healthcare facility where persons ... undergo rehabilitation to enable them to live independently at home," was an electronic whiteboard, a large device with good visibility. The whiteboard of the surgical suite is what Bossen et al. (2015) call the "common ground." This tool is essential to coordination and understanding between teammates. Situation awareness of the staff in the surgical suite relies partly on what is written on the whiteboard. It is a familiar tool that brings the benefits of pliancy allowing for quick creation and modification of the data. However, 
Branham et al. (2010) describe whiteboards as poor tools for archival and reuse, thus limiting their usability for analytics.

\subsection{Brief Review of OR Management Literature and Need for Real-Time Measurement System}

In his paper, Deming (1986) explains the principle of continuous improvement. He writes that a process is stable when performance variations remain within a regular range. His works show that measurement systems are important to assess the efficiency and bring organizations to a higher level of performance. Additional work on workflow analysis systems provided insight into the usefulness of assessment of new management rules, such as Rotondi et al. (1997), who put in place a real-time patient routing system. Although their system focuses only on the perioperative area and requires some manual data entry, they were able to assess the improvement through the implementation of multidisciplinary teams. Similar results have been found with systems following vital signs (Xiao et al. 2005) or following OR measurement systems like anesthesia management systems (Epstein et al. 2008; Lapierre et al. 1999). Some systems rely completely on Radio-Frequency IDentification (RFID), which is expensive and requires complex installation and tag deployment (Liu 2011). In our experience, technology adoption by medical staff is an important consideration, as some may be apprehensive of a system that tracks them (see also Prasad et al. (2012)). However, most of these systems except the RFID one perhaps required some manual entries to monitor the OR state. With this in mind, we designed the smartOR.

The domain of operations research tried to solve the issues of optimization of workflow in the OR suite. Guerriero and Guido (2011) summarize the work done in a detailed survey. They underline the power of mathematical modeling and the use of simulation to improve management of the operating theater. They emphasize the necessity to incorporate real-life aspects in future research such as individuals, seasonality, workforce capacity constraints, and scarce resource availability constraints (e.g., recovery beds, ICUs). The new challenge addressed in our work is a model that integrates knowledge from sociopsychology, industrial organization, and the surgical field to predict the dynamics of a complex organization (Forse et al. 2011). Urban et al. (2001) proposed a generic agent-based framework for human behavior, which is used in our work as a base for the construction of our model.

Over the course of the last 2 years, we have examined and tracked some of the behaviors and events in and around the OR using a set of three sensors (Garbey et al. 2015; Huang et al. 2016). Initially, we segmented out the events that occur during each operation in the OR, identified unique steps that would signal the initiation/cessation of each event, and created sensors that would target these automatically. For technology acceptance, it is essential to utilize sensors targeting tasks but not specific staff members. We did similar work in the area of minimally invasive surgery with the use of the smart trocar technology that has the capability to accurately record the time sequences of laparoscopic tool use (Toti et al. 2014).

\subsection{Technology Probes}

Probes are instruments allowing for the acquisition of unknown or incomplete data. Technology probes as defined by Hucthinson et al. (2003) have three dimensions: "the social science goal of collecting information about the use and the users of the technology in a real-world setting, the engineering goal of field-testing the technology, and the design goal of inspiring users and designers to think of new kinds of technology to support their needs and desires." We believe such a technology is relevant to be integrated in our MCPS because it supports the context awareness challenge mentioned by Lee et al. (2012) and Li et al. (2015), by using the interface as a sensor and 
cross-checking the shared representation of the situation the team has with the events detected by other sensors.

\section{METHODS}

\subsection{Architecture}

The cyber-physical system presented here has four components that work seamlessly together:

1. Inputs: The different sensors that acquire the information (sensors of the smartOR and BoardProbe)

2. Outputs: The network of interfaces that transmit information to the staff (including BoardProbe)

3. Artificial Intelligence: The mathematical model of the processes that delivers analyses and near-real-time feedback

4. The staff: The users and beneficiaries and thus the most important part of this human-inthe-loop system

Most cyber-physical systems would stop with these four components as they are sufficient to control the situation. For example, in the energy industry, a cyber-physical system of the power grid will rely on these four components to drive the switches and a human operator will expect to receive alerts from the system only in case of security breaches or hardware failures. However, the situation in an OR suite is more complex because multiple sources of information and collaboration of a transdisciplinary team are needed for decision making. Therefore, an additional component of the cyber-physical system interface is designed to efficiently share information and serve as a collaborative tool to the community of end-users. Furthermore, the staff of the hospital is focused on the usability and the value that the interface provides; full comprehension of the cyber-physical system or the constraints of any of its components is unnecessary.

\subsection{Methods and Constraints to Construction of the Sensor Components}

The philosophy behind our method is to promote technology acceptance by recording critical steps, not individuals, in the processes. In our experience, medical staff are often reluctant to be tracked by some form of RFID tag. Addressing this concern, we have focused our efforts on utilizing sensors that provide unbiased information on the OR state, as shown in Table 1. The system records precise information on when the patient is entering or exiting the OR, time at which the patient is placed under anesthesia, start and end time of the minimally invasive surgery portion, and other states. Utilization of three sensors is sufficient to efficiently reconstruct the OR state. However, caution is warranted in some situations, for example, living donor kidney transplantation, as tracked at one of our installations at the University Hospital of Strasbourg, during which the requirements might be more complex. In this scenario, additional time tags, such as time of the first incision and ischemic time (elapsed time when an organ or tissue is not supplied by blood), are necessary. Consequently, the MCPS must be modular to be able to add or remove sensors from the network at any time and provide alerts and redundancy mechanisms in case of failures.

In addition, sensors should require minimal maintenance. ORs are full of complex equipment and are carefully sterilized between procedures to prevent infections. It is particularly important to avoid battery-operated sensors requiring frequent maintenance, thus necessitating traffic in the OR, or additional sterilization of the sensor before each procedure.

For the cyber-physical sensors and network integration, it is mandatory to have a system that does not interfere with critical equipment of the hospital or increase the risk of a patient data leak. In the United States, any incident on patient data confidentiality is estimated to come at a cost of 
Table 1. Refined Array of Sensors Targeting Key Events at Each Step of the Procedure

\begin{tabular}{|c|c|c|}
\hline Step of Procedure & Event Target & Sensor Type \\
\hline Patient Brought Into OR & $\begin{array}{c}\text { Stretcher Crossing Door } \\
\text { Threshold }\end{array}$ & $\begin{array}{c}\text { Pressure-Sensing } \\
\text { Strip/Magnetometer }\end{array}$ \\
\hline Induction of Anesthesia & Ventilation Initiation & $\begin{array}{c}\text { Small Motion Detection } \\
\text { Camera }\end{array}$ \\
\hline $\begin{array}{c}\text { Laparoscopic Portion of } \\
\text { Procedure }\end{array}$ & OR Lights \\
Dimming/“Going Green” & $\begin{array}{c}\text { Small Motion Detection } \\
\text { Camera }\end{array}$ \\
\hline Reversal of Anesthesia & Ventilation Cessation & $\begin{array}{c}\text { Small Motion Detection } \\
\text { Camera }\end{array}$ \\
\hline Patient Exiting OR & Stretcher Crossing Door & $\begin{array}{c}\text { Pressure Sensing Strip/ } \\
\text { Threshold }\end{array}$ \\
\hline Occupancy of the OR & General Movement in OR & Infrared Motion Detector \\
\hline
\end{tabular}

\$2 million (Ponemon Institute Report 2014). Capturing procedural steps or OR states rather than patient data reduces the level of computer security required for the sensor network. Capturing patient information that is clearly independent of the EMR is a benefit of our system, reducing the concern for privacy issues. In addition, the internal processes and regulations of a hospital system may complicate the implementation of a system that presents a potential target for a cyber-attack. Anticipating the feedback of the medical team during the design phase is vital. In the OR, the medical team's priority is the patient, who might present with a traumatic and life-threatening condition. In such situations, a slight perturbation of routine through MCPS hardware would lead to, at best, reluctant adoption. Our system was developed jointly by computational scientists and the medical team on the project, with additional feedback from the stakeholders in the OR suite, and subjected to extensive testing prior to installation. In other words, the development was collaborative and dynamic.

\subsection{Understanding Staff Activity and Concerns}

It is of vital importance to understand staff activity and the user interface that serves as a collaborative tool. One of the human-machine research purposes here is to understand the surgical team activity and issues to develop a "user-friendly" system in our particular situation and, more importantly, provide guidelines to adapt the interface system to other surgical suites/other hospitals where the culture might be different. As we previously reported (Rambourg et al. 2016), this step is usually time intensive and therefore often skipped or done improperly in the industry. As this step is of the utmost importance for success, we use this opportunity to describe our investigation of user activity in the surgical suite in detail and present a set of guidelines for this human-in-the-loop system.

Currently, the central organizational and collaborative tool utilized for scheduling in the OR suite consists of two large whiteboards. The approach to capture the local activity in the OR suite involved two steps:

1. Contextual inquiries and shadowing of the main actors within the surgical suite

2. Capturing the activity and changes on the whiteboard with a camera

The conducted contextual inquiries involved direct observations within the surgical suite and interviews with two board runners, two surgeons, two nurses, two anesthesiologists, and a member 


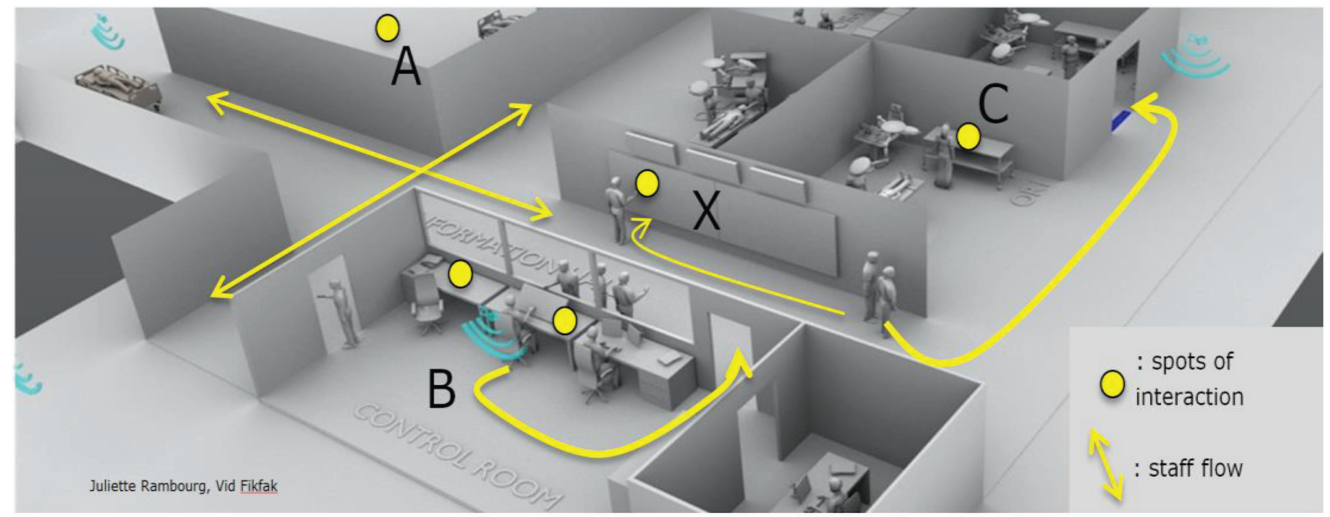

Fig. 1. Reconstruction of a surgical suite at Houston Methodist Hospital, with staff flow and interactive area of interest.

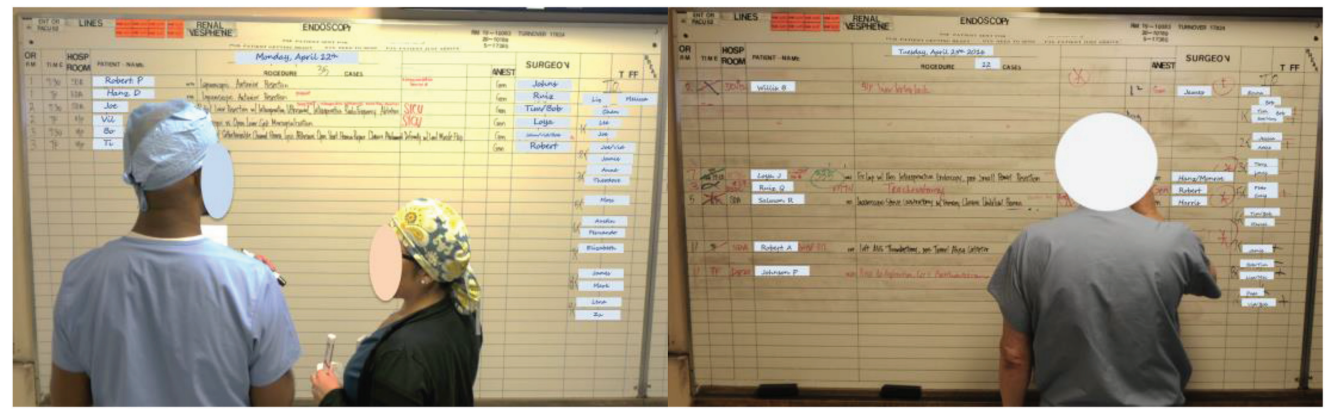

Fig. 2. Current scheduling whiteboard tool in use, in parallel with the hospital's EMR system. Patient and staff information is disguised.

of the surgical suite council. Due to the heavy workload, the interviews were conducted outside of regular working hours.

In Figure 1, "X" marks the location of the large whiteboard accessible and visible to the entire staff of the surgical suite. "A" marks the area of the pre- and postoperative rooms, and any additional rooms where the patient may be located before or after surgery. "B" labels the Interactive Area and " $\mathrm{C}$ " the ORs, where the surgeries take place. The large surgical suite at Houston Methodist Hospital contains 23 ORs, requiring very strict management of resources-human and equipment.

The scheduling, shifts, and patient location of all cases listed on the whiteboard are updated manually throughout the day as shown in Figure 2. All necessary information about the organization of the cases and OR suite management is contained on the whiteboard, making this a crucial and large source of information. The whiteboard allows for synchronous as well as asynchronous collaboration among the team, made up of board runners, nurses, surgeons, anesthesiologists, and members of the surgical suite council. The schedule for the next day is edited late in the evening on the whiteboard, ready for use by the staff early the next morning. Using a high-definition camera equipped with a large-angle lens, we captured 1,000 photos per day, taken with an acquisition frequency of 1 minute between each picture. The photos show the symbols written 


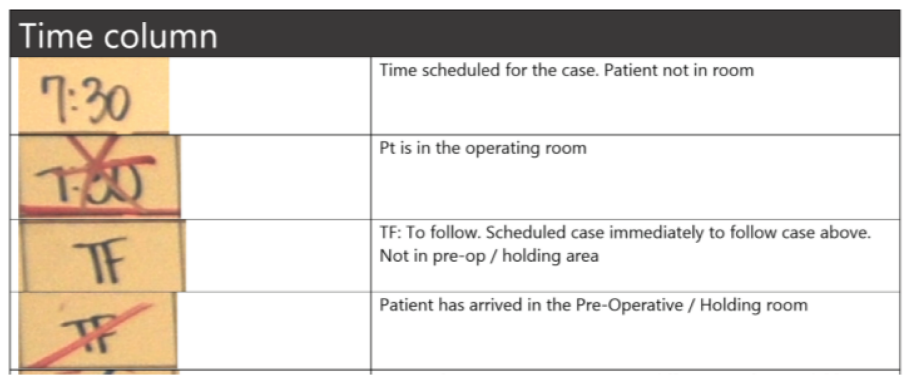

Fig. 3. Extract of the local dictionary used for the current scheduling whiteboard.

on the whiteboard, the modifications (updates, adds, writing, erasing) made throughout the day, and the user-whiteboard and user-user interactions.

From these studies, we extracted 20 identified scenarios of user activity involving the whiteboard. We identified 25 user requirements and more than 30 different "symbols" used by the team on the whiteboard. Figure 3 shows a part of this dictionary.

\subsection{Methods and Utilization of the User Interface as a Technology Probe}

The approach involves equipping the surgical suite information hall with BoardProbe, a whiteboard-like multitouch screen application, by following the Technology Probe design approach. The probe has the following three characteristics:

1. The social science goal of collecting information about the use and the users of the technology in operation and tracking how users interact with the probe over time. It is crucial to understand the local activity of the staff in the surgical suite.

2. The engineering goal of testing the technology in operation: the technology has to be functional and allow the user to perform daily tasks such as managing the schedule and adding and communicating information throughout the day. The aim is to employ this probe to measure efficiency and detect problems early.

3. The design goal of inspiring the staff and the design team to create features adapted to the needs of the surgical team.

The design methods of the user interface consist of an end-user-centric loop. At each iteration of the design, from low-accuracy to high-accuracy prototypes, the interface was tested with a team of end-users. At first, we used paper prototypes to simulate software use on a multitouch screen. The different elements can be moved, touched, swiped, and so forth easily as shown in Figure 4.

For further development, multitouch screens were utilized. The users, participating as designers, are engaged and invested in the project, allowing us to capture their interaction with the interface and generate additional ideas for desirable features.

\subsection{A Workflow Modeling}

Arising from the conceptual work described above on the MCPS, a mathematical model of the surgical flow in a large OR suite emerged. Each OR process can be represented by an algorithm that has a fairly complex graph structure for its execution and requires a minimum team of players to achieve the task corresponding to each node. Normalization of large-volume surgery, such as cholecystectomy $(600,000$ cases per year in the United States) or bariatric surgery $(200,000$ cases 


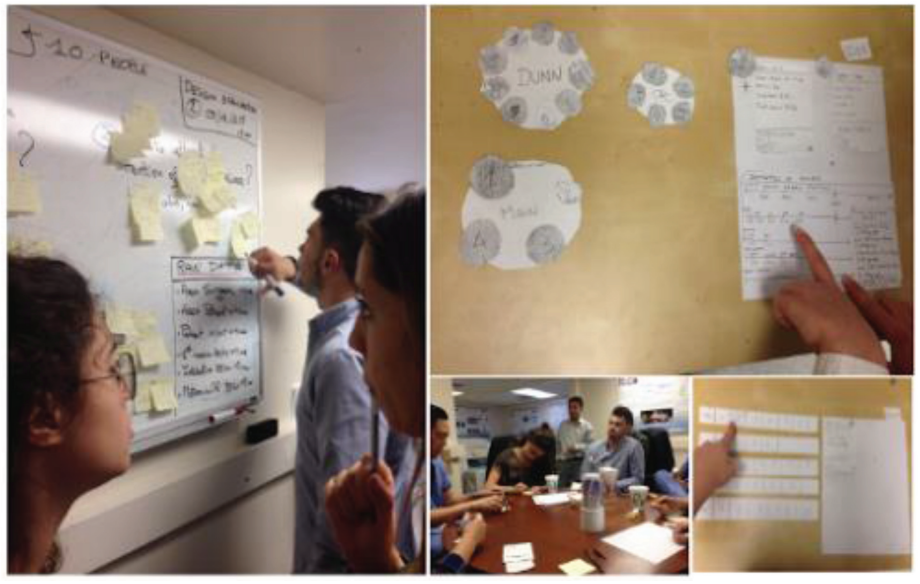

Fig. 4. Participatory design session.

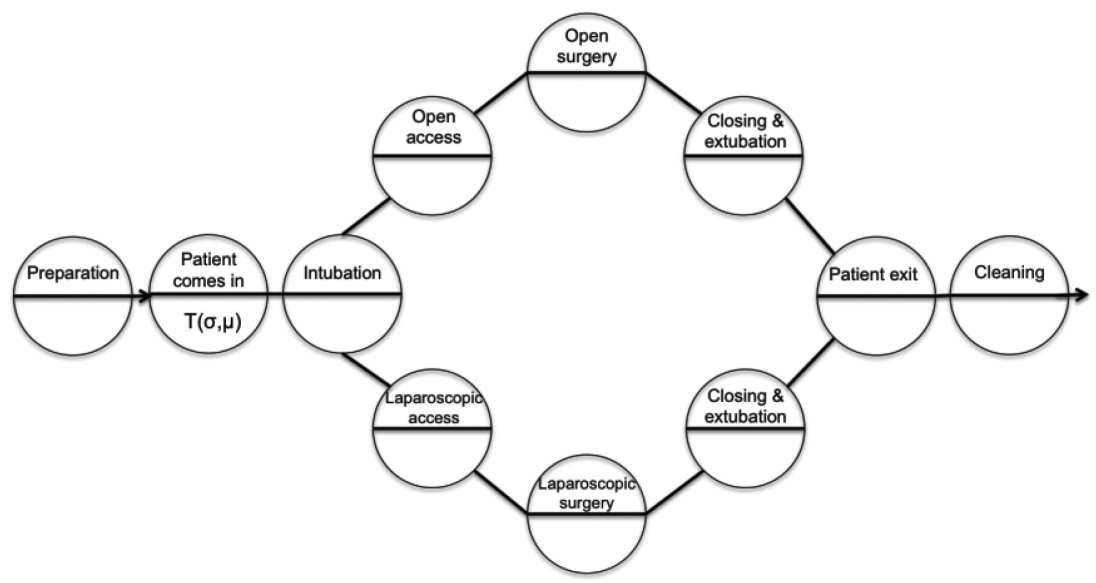

Fig. 5. Example of surgical flow for the two most common types of surgery in each OR with the task progression from left to right. The transition from one box to another takes into account probability distribution of time found with the analysis of our clinical data.

per year) by major surgical associations such as the Society of American Gastrointestinal and Endoscopic Surgeons (SAGES) provides a solid base to develop a model.

Our analytical method results in a staff-specific, agent-based model that retains the key features observed in daily clinical practice and is based on a comprehensive multiscale view of activities in the OR suite. The goal of the mathematical model is to add some predictive simulation capability of the surgical flow to help optimize OR management decisions. The proposed model explicitly takes the OR staff and their communicational and technical skill levels into account. Parameter identification in the agent-based model improved as our database of smartOR data grew and became patient and surgeon specific, by relating it to the EMR. For example, once the timelines of hundreds of open surgeries and MIS are available, a good statistical predictor of the time spent at any given step of the process can be developed as shown in Figure 5. The preliminary analysis was able to differentiate between standard and longer cases as a function of patient risk class 
Table 2. Ranking the Influence of the Parameters on Key Factors of OR Efficiency, Such as Turnover

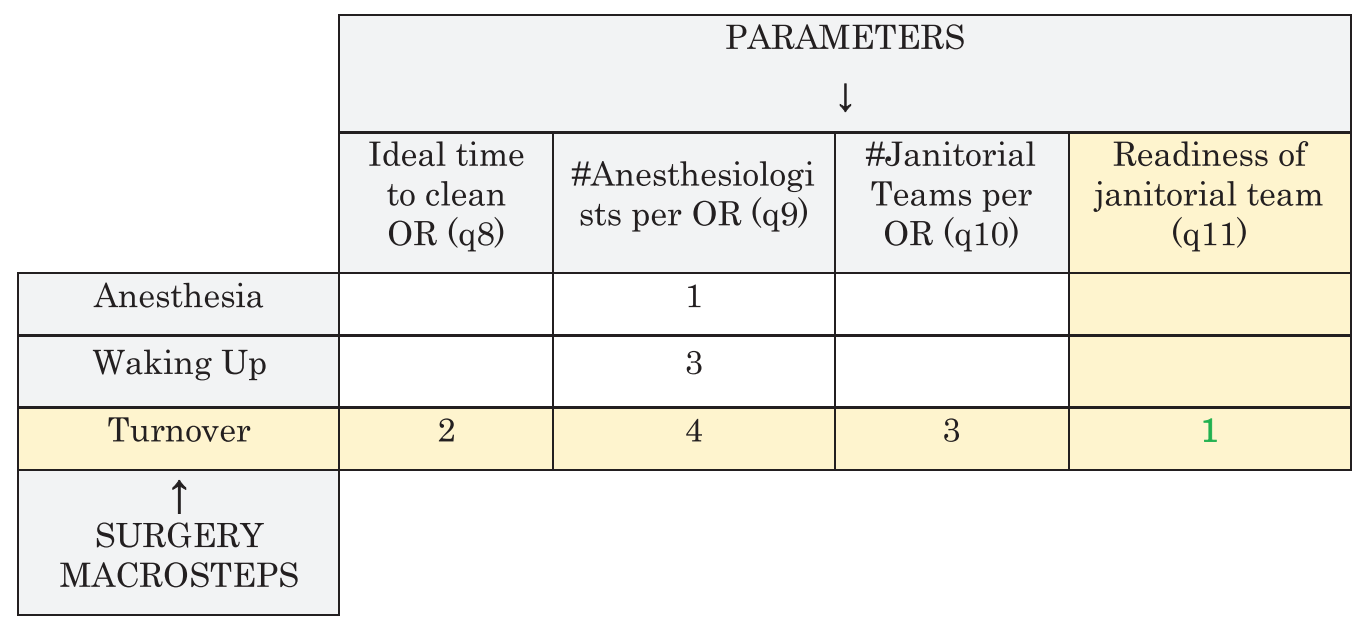

in anesthesia (e.g., bariatric surgeries) but not yet at a satisfactory level of precision. Of course, the patient condition prior to surgery plays a key role in those predictions and needs to be much further refined in the analysis. Therefore, to provide reliable guidance, the MCPS must be able to recover extensive information from the EMR system of the hospital. This critical step will be further discussed in Section 5.

Here, we discuss how the model accounts for the awareness factor; a more detailed description was previously published (Garbey et al 2017). The model ranks the influence of parameters, such as the ideal length of time to clean the OR or the number of anesthesiologists/number of ORs, according to their impact on the macro steps of the procedures (turnover time, anesthesia time, etc.); see Table 2. One (1) is the highest ranking (i.e., it has a very high impact on the macro step performance), and four (4) is the lowest impact score. Parameter q11, awareness and communication delay until a janitorial team arrives in the OR, has a very high impact score (1) on the turnover time performance. Therefore, the model suggests that we must increase our efforts on improving surgical suite awareness to improve turnover times. This underscores the importance of deploying a smartOR/BoardProbe system that can reduce delays and thus turnover time.

Finally, and very importantly, verification and validation are an essential part of the design cycle. Verification ensures that the software correctly executes the task; for example, if a sensor is supposed to catch when the patient is coming in or out of the OR, there should be no false-positive identification of that event that corresponds to another type of traffic. Verification ensures that the analysis of the sensor output can separate common events such as "staff or light equipment coming in and out" from the "patient bed with the patient entering or leaving the OR." Validation would correspond to the fact that the MCPS provides correct guidance on OR management as a result of the mathematical model. Both verification and validation end each design cycle of our implementation and allow for the next improvement of the system through a new design cycle.

\section{CPS PRESENTATION: THE CYBER-PHYSICAL INTELLIGENT OR SYSTEM}

\subsection{Capturing Operating Room Status and More}

As previously reported, the system has been active for over 2 years in multiple ORs, where several types of general surgeries are done (Huang et al. 2017). Here we discuss the three key sensors 


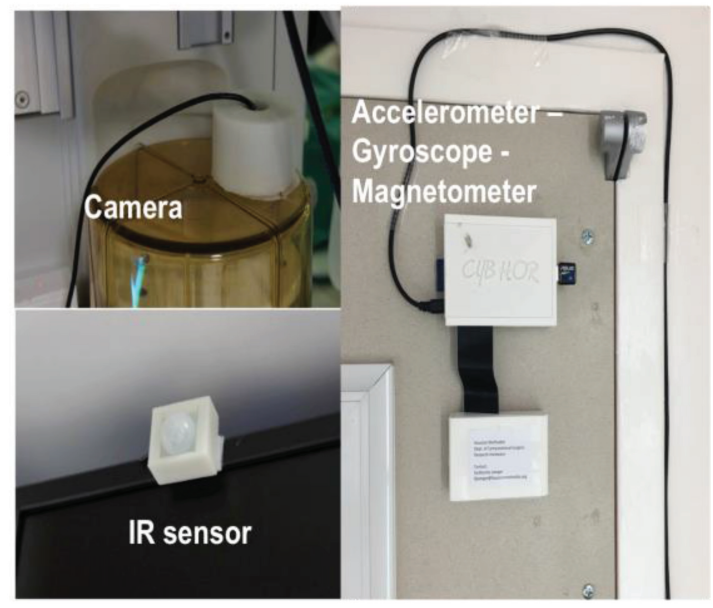

Fig. 6. The three sensors installed in an OR. The camera is placed on top of the ventilator bellows using a 3D printed case (top left). One of the corners of the room contains the IR sensor (bottom left) and the nine-degree-of-freedom sensor is on the door (right).

installed at key points of the ORs and which allow for the detection of all these different cases and their respective steps. A simple InfraRed (IR) detector with a very large angle of view detects the presence of personnel in the room. Detecting the entry and exit of a patient was challenging. We started initially with two parallel pressure strips embedded in mats placed at the doorstep, but had to improve that sensor through multiple design cycles because the standard of the shelf pressure strips available on the market was insufficient to hold the pressure of a 400-pound rolling bed. In addition, it was determined that the presence of the mats at the doorstep was burdensome to the cleaning crew. Attaching an accelerometer-gyroscope-magnetometer to the door provided a satisfactory solution. This also not only provides information on the traffic of the patient bed through the signal generated by the magnetometer but also is useful to provide data on the overall traffic at the door. This incidental feature is significant in light of the finding that OR traffic in and out may have an influence on infection rate (Andersson et al. 2012). Finally, a camera mounted on the ventilator of the anesthesia machine (see Figure 6), directed down to detect the motion of the bellows, obtains information on intubation/extubation of the patient. This sensor also provides information on the laparoscopy time in case of minimally invasive abdominal surgery as it detects changes in light condition such as dimming the luminosity and using green lights in order to help the surgeon work with better contrast on the endoscope view.

It must be emphasized that each of these three sensors is connected to electric wall plugs and does not require sterilization. Our hospital management crew was also encouraged by the fact that it was possible to recover OR stages with only three low-cost sensors placed at locations that did not interfere with the surgical team's routine.

The same pragmatic approach was utilized to add sensors on demand depending on surgery need. Each sensor has been designed to detect one or more events and has custom Wi-Fi communication protocols for wireless transmission and ease of installation. All sensors are connected to a commercial single-board computer. The devices communicate with a central computer using a secure TCP network. Each sensor has its own name and identification (ID) code. When communicating with the central computer, each sensor sent its ID tag, the present time, and a digital output of the sensor measure. This information can be encrypted. Identifying the event from the sensor 
output requires some specific signal processing either to clean the signal or to identify a change of state. In addition, information should be put in the context of the past time series of all sensors for that room to ensure the analysis is correct. To avoid the detection of false positives, real-time detection cannot be implemented and the polling update frequency is set to 2 minutes. The raw data for potential a posteriori analysis and verification purposes as well as the event detection output, which is a simple discrete time, are saved. All the algorithms on the host and the client are written in Python. The detailed unbiased information that the smartOR output combined with the EMR provides is invaluable since it enables OR management to get the level of trusted, fine-detailed data required for efficient and informed management. In addition, this MCPS also has the capability to capture critical information that relates the activity of the OR to the peripheral area. The system presented here is robust to detection of any kind of open or MIS surgeries done in ORs where general surgeries are performed. The information generated allows two kinds of analysis: one focusing on the general efficiency of the OR taking into account any type of surgery and the second one on the workflow of specific surgeries such as only laparoscopic ones or, even more specific, bariatric surgeries. However, for endoscopic surgeries or others that do not utilize the automatic ventilation, the bellow movement does not initiate and thus the system does not register this step. Current work includes a system that could address these surgeries and will be soon tested in a GI suite for validation.

It was noted during the first development phase that delay in the first surgery of the day was a very important factor impacting subsequent operations in the OR for the remainder of the day. It is standard practice that the surgeon meets his or her patient in the preparation room immediately before the patient is transported to the OR. To objectively record that time point, we installed a minimum RFID setup that recorded the time when the surgeon was at the doorstep of the preparation room. We found that the surgeons visit the patient on average 8 minutes before the scheduled starting time, with $69 \%$ of the first cases starting late; see results in Huang et al. (2016).

\subsection{Displaying and Capturing Information}

Our work utilizes three different levels of human-technology interfaces:

1. Local: Each entry to an OR needs an interface to display quick and simple information.

2. Protected: The control room is the central collaborative and informative area and thus our initial target to provide information.

3. Public: The information hall, the location of the traditional whiteboard, is where personnel exchange information with the rest of the staff. BoardProbe supports this interface.

\section{Operating Room Door Interface}

The system needs to have different levels of dissemination of information. While the control room is the central point, not everyone has access to this room, its documents, and computers. Therefore, it is necessary to have a local point of information dissemination such as the OR entry. As shown in Figure 7, each OR should have its own information screen adjacent to the door displaying the current status of the room with an easy three-color code indicating the successful-or delayed-progress of the operation.

\section{Control Room Workstation Interface}

The current management of our OR suite employs an operating room coordinator (ORC). The ORC uses the whiteboard in the hall of the OR suite and spends a significant amount of time acquiring missing information and contacting personnel in an attempt to resolve inefficiencies. The first step in delivering the MCPS project was to develop a multitouch application on a 42 " screen that allows the ORC to work quietly on a workstation in the control room. The system is designed to display 

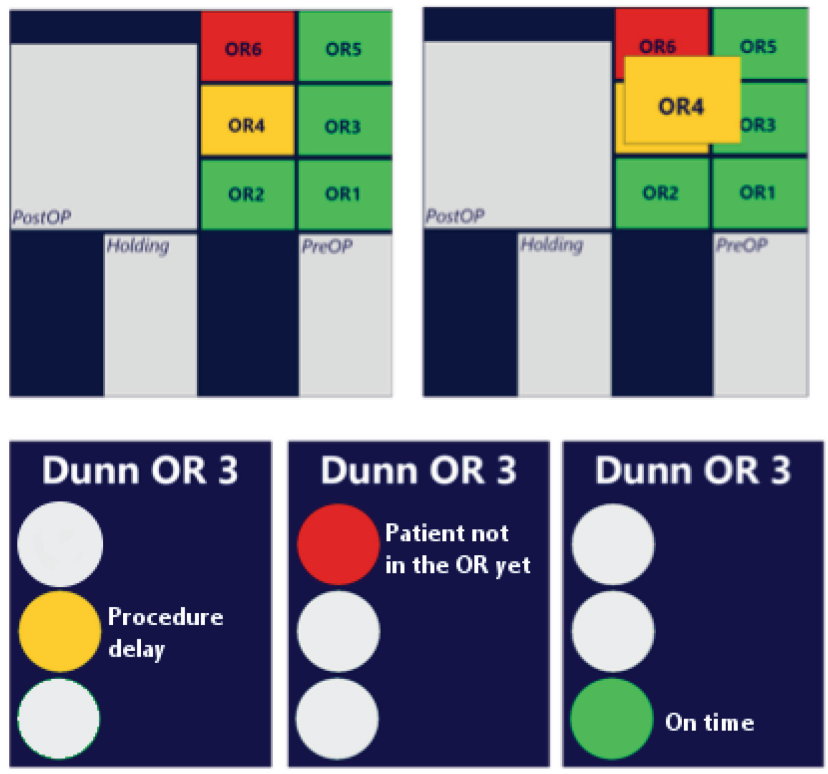

Fig. 7. Two levels of disseminating OR status messages: global overview of all the cases (top) and local status of cases (bottom).

the OR status, the location of patients and surgeons in real time, and specific alerts, as shown in Figure 8. Based on OR staff feedback, we defined "near real time" for the purposes of this article as an information update at least once every 2 minutes.

The main work of this project focused on building a Human-Computer Interface that requires very limited user training. The interface displays information in a way familiar to the end-user through a unique, annotated, and simplified map of the surgical suite, and limits the use of graphics or tables, thus making it easy for the end-user to navigate. The Graphic User Interface on the workstation was codeveloped with some of the medical staff and evaluated by others through a rigorous protocol. We followed a user-centric, iterative design protocol: the interface was developed first at our facility after contextual interviews and observations of the surgical staff. Subsequent usability tests detected mistakes or nonusable features. To close the design process, the same tests were performed on a new version of the software at the target location of the hospital with the staff. The staff must be emancipated from the complexity of technologies so that the surgical team can focus on the patient. Improving staff efficiency leads to a better use of the facility (for instance, reducing the idle time of an OR) and therefore possibly results in increased OR utilization.

\section{BoardProbe: A Technology Probe for the Surgical Suite}

The main challenge when computerizing any activity is to achieve a smooth transition to new technology under operational and critical conditions, and BoardProbe appears to be a suitable substitute for the current whiteboard in use by the staff. It allows the surgical team to keep its focus on the patients, not on the technological changes. Moreover, staff member learning and acceptance of technology vary.

The whiteboard, shown in Figure 2, lists all cases scheduled for the day and throughout the day tracks all changes pertaining to patients, staff, and equipment. In order to increase technology acceptance among the staff, it is beneficial to have the new tools closely resemble former ones while 


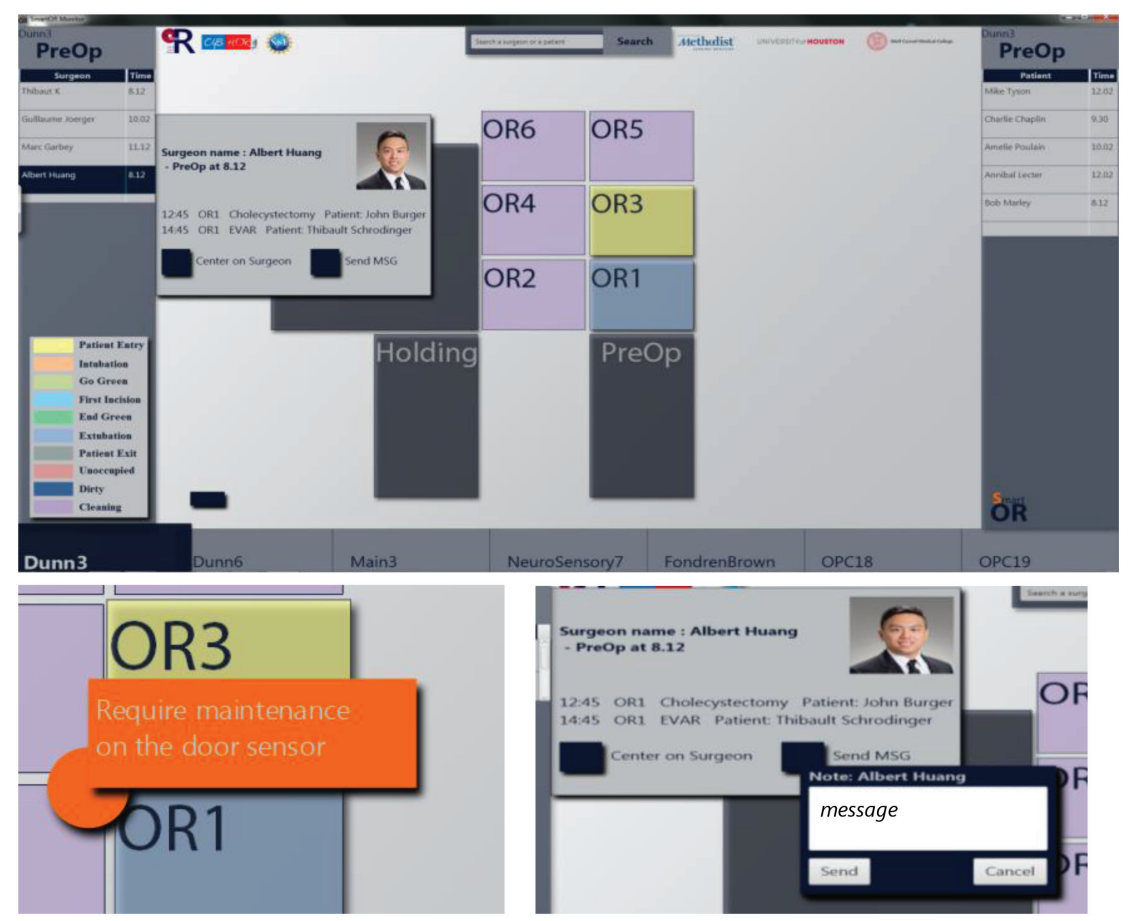

Fig. 8. Control room workstation interface.

adding improvements. The technology presented the staff with the exact same way of working: a whiteboard, but electronic this time, on which they are able to write and erase, thus minimizing the disruption of the arrival of a new tool. The electronic interface also offered the opportunity to test new features with the users that proved useful in terms of time and visual efficiency. Engagement of the end-user opened the opportunity to receive additional end-user requests and customize the tool with additional needed features.

Figure 9 shows the one-page application referred to as BoardProbe. It consists of an electronic whiteboard and electromagnets on which the staff can easily write or erase. Items can easily be moved on the multitouch screen that in size and layout is as close as possible to the actual size of the whiteboard currently used in the surgical suite. To allow accurate manual writing, the tool has high resolution and provides the flexibility experienced on the traditional whiteboard. Pliancy is of high importance to the system not only to fit the constant changes as well as anticipate any new scenario of use but also because BoardProbe is a technology probe, meaning that its use provides a way to capture and understand the user activity better through logs of the inputs. This is why we allow write-erase interaction everywhere, as well as blank magnets. Below are the details on the three dimensions of BoardProbe:

1. BoardProbe's visualization part corresponds to the common awareness of the staff in regards to the status of the cases in the surgical suite. The staff common awareness is also spread throughout the hospital as some staff members are not in the surgical suite but need information that is on this whiteboard. It allows scheduling, like the traditional whiteboard, and it brings more up-to-date information from the smartOR sensors. It is not enough that a few people are informed of the current status, and the smartOR sensors and the inputs 


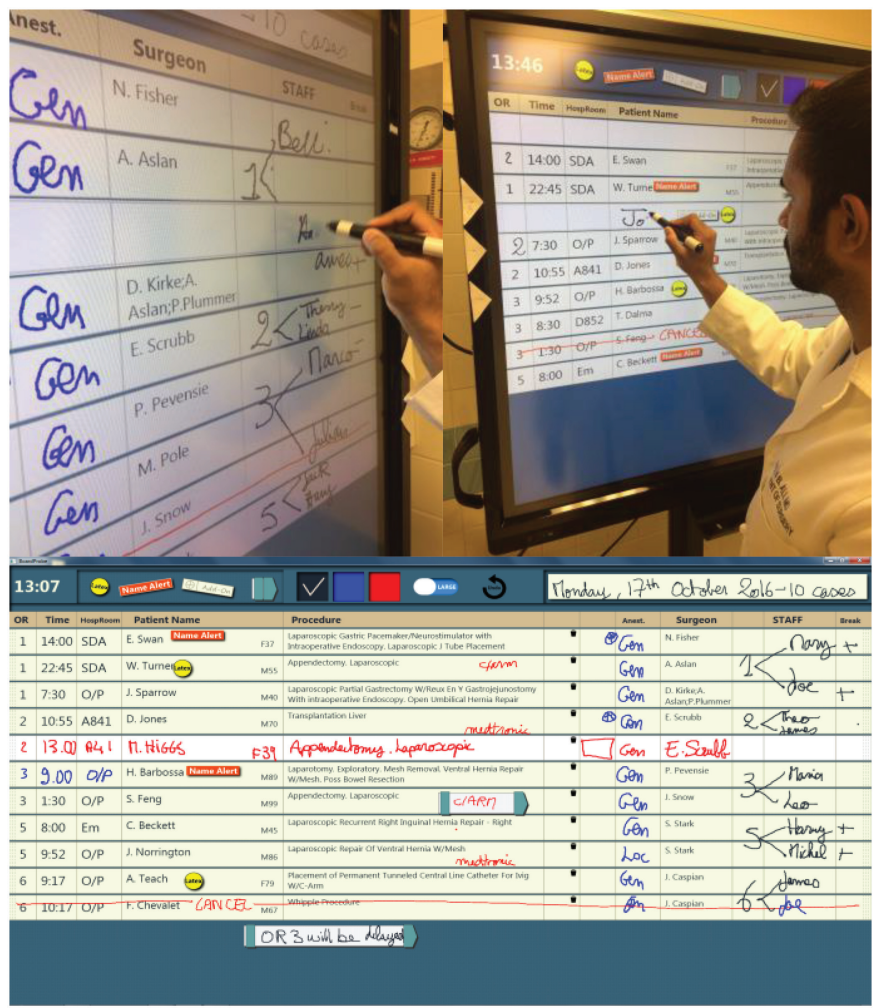

Fig. 9. BoardProbe: A technology probe for the surgical suite (fictitious names shown).

made on BoardProbe allow for the display of information to other locations throughout the hospital. BoardProbe will display the near-real-time status of the surgery: Room empty Patient enters the OR - Patient intubated - Patient extubated - Patient leaves the OR. In this way, the operator can visualize this important information at the same time that he or she is planning scheduling/rescheduling. It will allow management to detect and anticipate inefficiencies. For instance, BoardProbe shows that the OR is still empty although the next surgery was scheduled at that time; therefore, the staff can be alerted of the delay more in advance and find an alternative. As another example, the OR status shows that the patient exited the OR, and thus the janitorial team knows that the OR can be cleaned as soon as possible.

2. BoardProbe's technology probe part allows us to capture user activity and understand their needs better. The triple interest is to collect information about the use over time, test the technology in operational conditions, and inspire the staff and the design team to create additional features.

3. BoardProbe's sensor part brings an additional type of data in near real time to the database. Detecting what is added on the schedule and distributing the information to stakeholders of the surgical suite or even of the whole hospital is an identified need. The following is a scenario reported by the MD team: Dr. Smith is a resident in surgery; he has surgeries that are planned throughout the day but he can never be $100 \%$ sure of when they start, since start times depend on the end time of prior surgeries. However, Dr. Smith needs to work in other 
Table 3. Results of the Users on the Main Factors of Efficiency on BoardProbe Usability Test

\begin{tabular}{|c|c|c|c|c|c|}
\hline $\begin{array}{c}\text { Task } \\
\text { Duration }\end{array}$ & $\begin{array}{c}\text { Learning } \\
\text { Time }\end{array}$ & $\begin{array}{c}\text { \#Failed } \\
\text { Commands }\end{array}$ & $\begin{array}{c}\text { \#Help } \\
\text { Requests }\end{array}$ & $\begin{array}{c}\text { \#Time- } \\
\text { Consuming } \\
\text { Commands }\end{array}$ & $\begin{array}{c}\text { Time Spent on } \\
\text { Nonproductive } \\
\text { Activities }\end{array}$ \\
\hline $\begin{array}{c}10 \text { ' to } \\
2 \text { '41" }\end{array}$ & $2-5$, & 5 & 0 & 1 & 0 \\
\hline
\end{tabular}

parts of the hospital before and after his cases. He cannot physically watch the whiteboard. Therefore, Dr. Smith has asked coworkers to watch the whiteboard for him and inform him whether the previous case is over or not. Therefore, spreading the information written on this whiteboard electronically has the potential to save resources.

The evaluation of BoardProbe as a user interface and sensor has undergone a first stage of pure usability tests, without sensor connections. Two board runners volunteered to participate in a 30minute usability test on the system including a set of 10 tasks corresponding to their daily activity on the whiteboard involving collaboration or not, decision making, and update of the schedule. Three main aspects of usability were included: effectiveness, efficiency, and user satisfaction:

Effectiveness: All usual whiteboard tasks were performed using BoardProbe. The participants used $100 \%$ of BoardProbe's features, with a success/failure ratio of $95 \%$.

Efficiency: Quantified through specific factors that give us an indication on the quality of the performance, as shown in Table 3.

User Satisfaction: The System Usability Scale (SUS, by Brooke (1996)) results give an average score of 80. Bangor et al. (2009) consider that a prototype with a SUS score of 80 is "Acceptable" in the acceptability ranges, graded between $\mathrm{C}$ and B and halfway between the adjective rating "good" and "excellent."

This is the first set of usability tests in a controlled environment and we are now working on getting the authorization for clinical trials. In our effort to integrate this system in the real world for clinical evaluation, we determined the high-impact factors that will make the difference. In order to make the staff accept the technology chosen by the designers of the system, we suggest that three critical points must be taken into account:

1. The quality of the hardware (Fitzpatrick et al. (2013)). The hardware can be a deterrent to adoption if it is not reactive enough. For the purpose of the electronic whiteboard, the use of PCAP (Projected CAPacitive touch) or InGlass technology is recommended to accommodate any work that has to be done on multitouch applications - IR technology unfortunately detects the touch slightly before the user touches the screen, potentially creating false inputs.

2. The constant involvement of the surgical suite staff in the conception process is absolutely necessary to ensure that the system suits their needs: Stakeholders are often very enthusiastic and enjoy being involved (at least in our experience at our home institution) in the design of a new tool they would use in the clinic, if they understand a direct potential impact on how the tool will help them.

3. The scalability of the system: Each surgical suite has commonalities, but each has its own "culture," habits, and needs. Conceptors, therefore, need to understand the essence of the system and have software and hardware pliant enough to support the activity in different environments. 


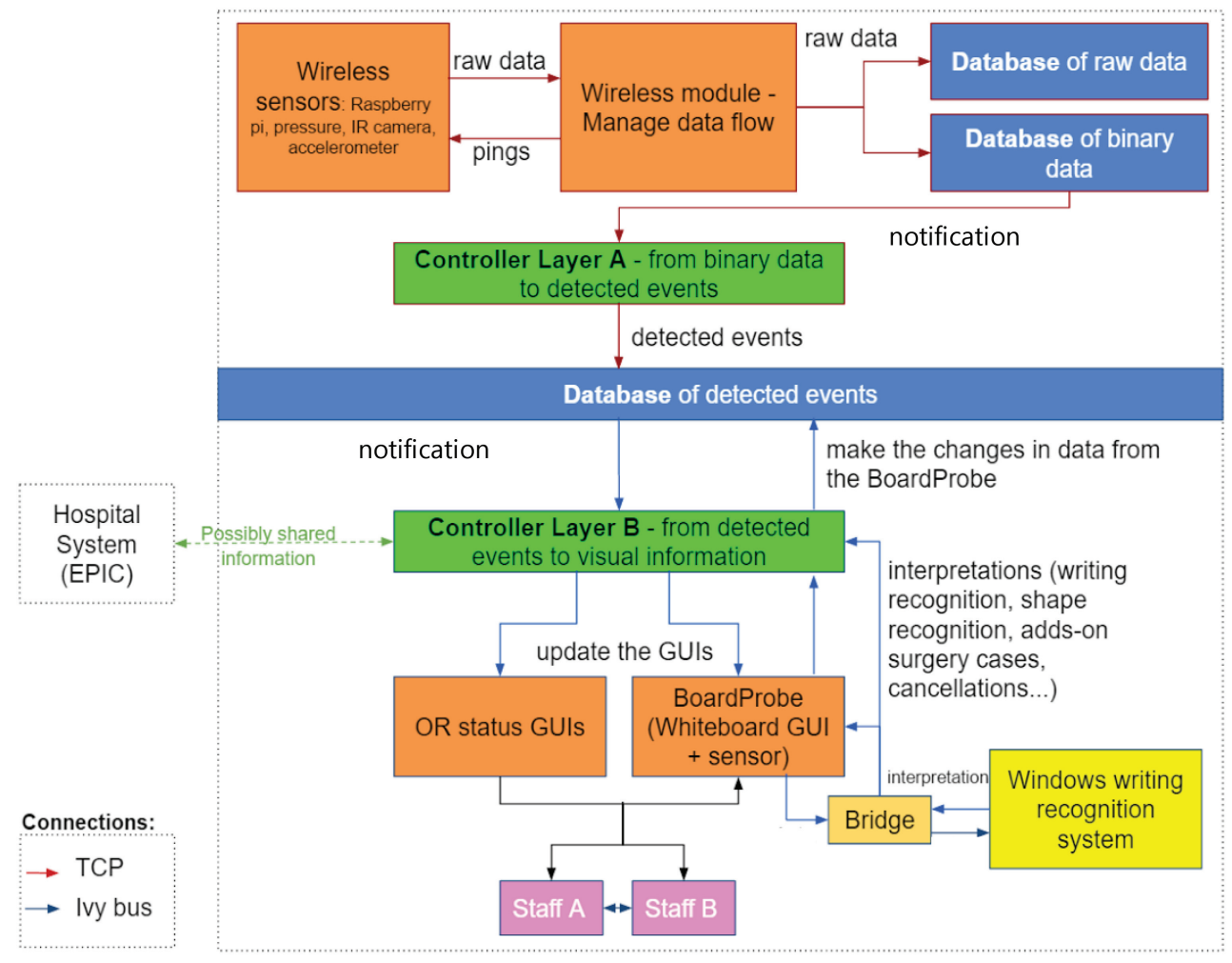

Fig. 10. Architecture of the system.

Analyzing the activity from the BoardProbe such as tracking written records and use of icons and cross-checking with the smartOR sensors allows for the detection of errors or singular events and delivery of an early alert of the problems. This adds another dimension and valuable feature made possible by the MCPS.

\subsection{The smartOR-BoardProbe link}

The cyber-physical system we designed and built, described in Figure 10, is composed of a cluster of simple but carefully selected technologies working in sync. Integration of multiple technologies allowed for the creation of a network accepted by staff and integrated within the surgical suite. The smartOR system has been in use for almost 2 years in two ORs and an additional four ORs went online recently via another $4 \mathrm{G}$ mobile hotspot. Expansion beyond the initial system is pending the hospital's Internet access authorization. These sensors provide an efficient, user-centric, unbiased, and noninvasive method that delivers critical information to the staff. The smartOR sensors automatically record key information from the OR that was previously unknown to the hospital or that had to be manually entered. BoardProbe saves key information from staff inputs for collaboration and planning that was previously not recorded at the same time as it delivers information.

Adding the BoardProbe capabilities to the smartOR is similar to adding a new sensor to the system and allows for the comparison of staff common awareness to OR state reality. A first example of cross-checking information would be the end of a case: when the patient leaves the OR, the ORC crosses the corresponding line on the electronic whiteboard. With the electronic board, we can detect this writing and then check whether the patient did leave the OR through the smartOR 

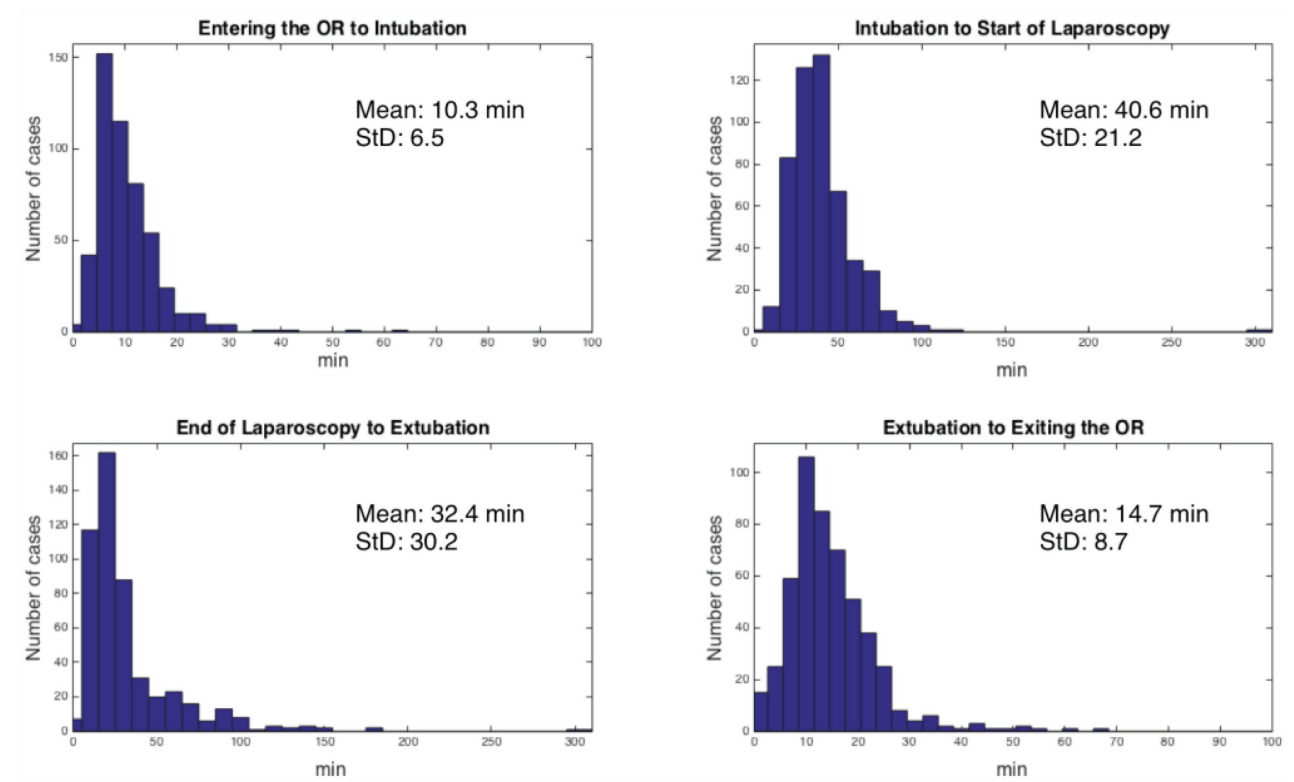

Fig. 11. Elapsed time distribution between the different stages of the surgery the smartOR can detect with respective mean and standard deviation. The cases longer than the mean plus one standard deviation (StD) are considered suboptimal and constitute $38 \%$ of all cases.

sensors, and if not, an alert would display. This example would prevent a scenario where the staff believes the patient has been moved from the OR while, in reality, the patient is still in the room.

Our smart OR records shows that in $11 \%$ of the cases, the patient remains in the OR even though the case has concluded and the patient should have been moved to the postoperative area; see Figure 11 (bottom right plot). This is frequently because space in the postoperative area is limited and the patient cannot be accommodated.

\section{RESULTS AND LESSONS LEARNED}

This MCPS implementation was pain-free and resulted in transparent daily production of time series describing several aspects of the surgical flow in the surgical suite that can be used in the OR management board meeting.

Between November 2014 and December 2015, data on more than 1,000 cases from two ORs was collected and analyzed. Half of them were minimally invasive or laparoscopic surgeries; the rest are a combination of general surgery, vascular, plastics, neurosurgery, and colorectal procedures. As the smartOR system is capable of segmenting the OR stages as well as discriminating an open surgery from a laparoscopic one, we identified and analyzed 504 laparoscopic cases segmented into five parts of the OR cycle: time to intubation, time to access, laparoscopy time, time to extubation, and time to leave the OR; see Figure 11. Suboptimal was defined as any elapsed time being longer than the mean plus 1 standard deviation. Figure 11 shows that on average, cases are suboptimal in $11 \%$ of cases for each step, and $38 \%$ of all cases are suboptimal in at least one step; refer to Huang et al. (2017) for more results. This shows that sources of inefficiencies may come from any time segment of the surgery cycle. Turnover, one of the main metrics in OR efficiency used by hospital management, was also computed. Figure 13 shows that turnover time was frequently over the 30minute standard proposed by the hospital and that sources of inefficiencies may come from any 

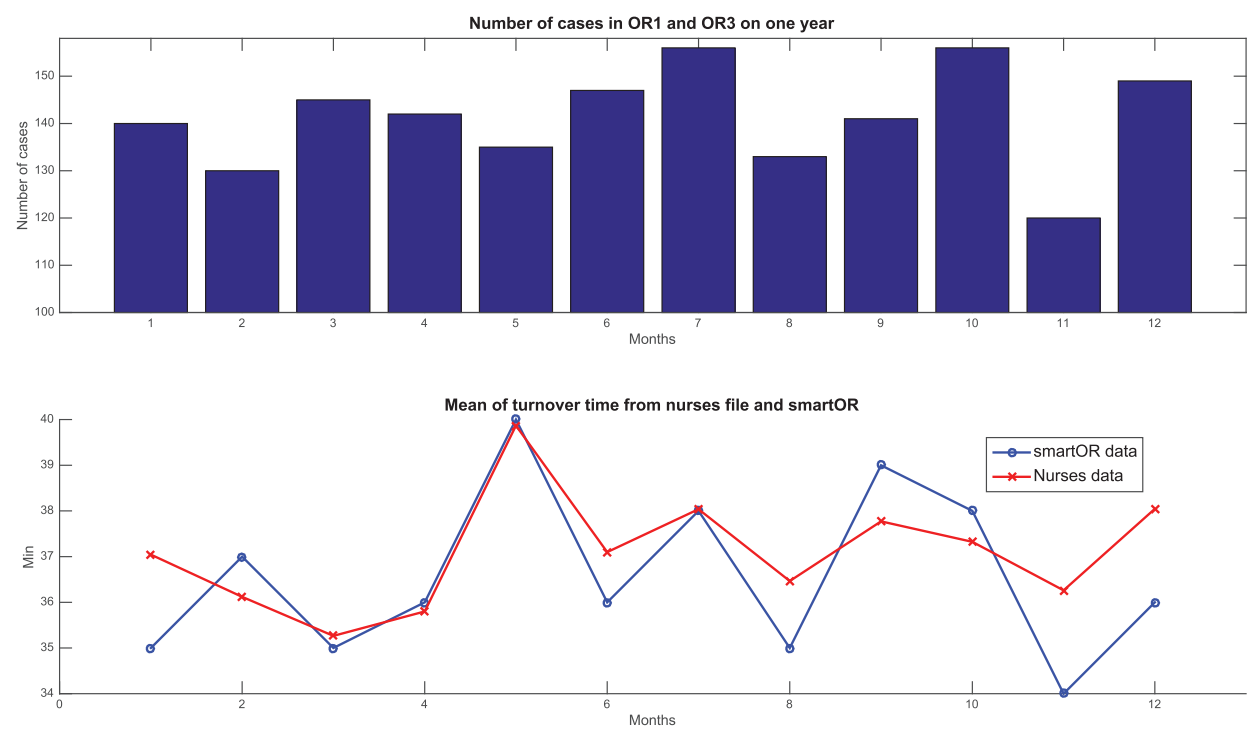

Fig. 12. Comparison between the mean turnover time reported by the nurses (red) and by the smartOR (blue). Length of turnover time has no correlation with the volume of cases.

time segment of the surgery cycle. Nowadays such basic information is not commonly available in real time and thus the management is unable to adapt OR allocation as a function of delays.

When building a system that is going to be installed and used every day in the most complex place of a hospital, accuracy and robustness are keys for acceptance and utilization. Our system relies on the accuracy provided by the different off-the-shelf parts used, and thus validating both the hardware and the software was crucial. The first step after installing the system in the clinical world was to compare sensor-triggered events with documentation from the nursing staff. In comparing the smartOR acquired times at which the patient entered the room and exited the room to the nurse's manual entry time, the Pearson coefficient was 0.8 , indicating that there was a consistent positive correlation between the sensor-acquired data and what was manually entered into the medical record. Figure 12 reports on the average turnover time, i.e., elapsed time between two consecutive surgeries, of surgery cases during a 1-year period that compares the smartOR output data with data entered manually by the nurses on duty. We were able to verify that not only was the smartOR output correct but also the time entered by the nurse was often delayed. Although there is not a large trend, we can see that in general the elapsed times coming from our system are shorter than theirs and thus are more optimistic than theirs, as you want the turnover time to be as short as possible. This is probably linked to the fact that the EMR chart is usually filled after the events when the staff have time to write it down, which introduces some level of estimation and error to the data. We have the same result on the average start time of the first case of the day. Another observation is that longer turnover time, as well as delayed start times, do not correlate with the volume and complexity of surgeries scheduled for the day. Overall, analysis of OR flow is not straightforward and one must consider many diverse factors.

In Joerger et al. (2017a), we quantify the delays between the detected events, for instance, the difference between when the patient actually enters the OR and when it is reported on the whiteboard (15 minutes of delay with a standard deviation of 22 minutes). The whiteboard constitutes the "common ground," the common awareness of the team (Bossen et al. 2015). Such delays make 


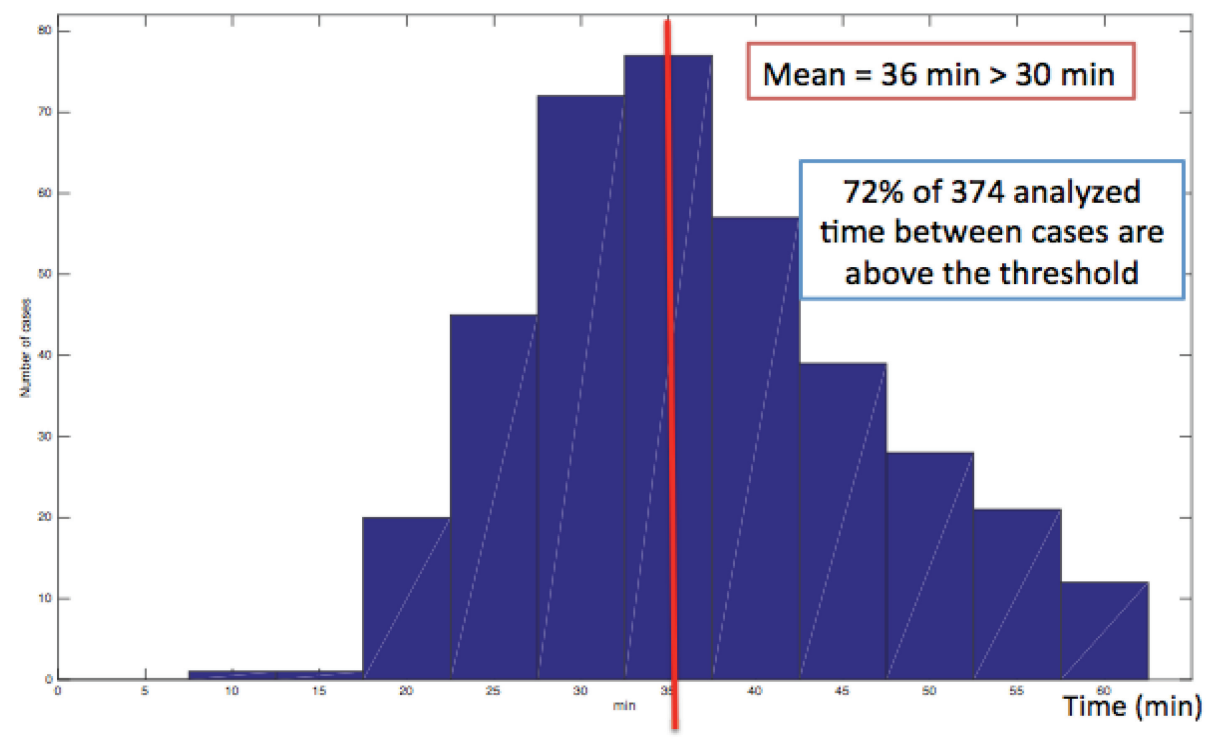

Fig. 13. Distribution of turnover time.

near-real-time management of patient flow much harder. With the link between smartOR and BoardProbe, these delays will be brought to a near-real-time update. A better awareness of OR status will bring more confidence from the staff in the system, with fewer workarounds that can compromise safety such as interpretations, deviations, and improvisations (Debono et al. 2013), and therefore will make the scheduling and collaboration activities safer, more reliable, and easier.

The next step is to generate a statistical model of each surgery type. It is intuitive that larger databases of surgical records will increase the accuracy of the model.

However, additional factors play a major role in the progression of a surgery. Patient condition and health history are important considerations. To ensure an accurate and feasible model, it is therefore inevitable to modify the statistical model to accept information from the EMR. This requirement adds additional complexity because the linkage between the MCPS and EMR database has both technological and legal challenges. While we have thus far been unable to make this connection, we are hopeful that our preliminary results in bariatric surgery will provide convincing results to show that OR time allocation can be dramatically improved with this information.

The second lesson learned was that getting OR staff engaged in the design process improved technology acceptance, and the feedback improved the technology itself as well. Many times the ideas generated in our lab did not pass the reality-check test under clinical conditions by the end-user. As soon as the OR staff realized that their feedback was valued and incorporated, the buy-in increased and the project vastly benefited from it. Consequently, it is important to note that the MCPS should take into account the local workflow established by the OR suite crew. Not only may different hospital systems have different practices, but also it is even common that locations within the same hospital system may differ. This lack of standardization is very different from other industries such as manufacturing or service industries, which have undergone a very rigorous standardization of processes. A good example to illustrate this issue is the observation of the whiteboard activity: one can expect that vocabulary and notations are so important to build OR awareness and evolve with time and practice. OR practice changes very quickly with new imaging or robotic tools that are emerging and evolving very rapidly in the surgical word. The MCPS 
should have, then, the flexibility to incorporate these changes. Once again, the engagement of OR staff in this constant evolution of the design might be the recipe for success, but the electronic board can be seen as a means to capture that evolution and discuss it with the end-users.

A third lesson was the necessity to make every effort to smoothly transition from the current state of the OR suite managed by the whiteboard to an electronic board that integrates every aspect. Once this step was achieved, it gave an opportunity to the end-users to propose new services that would not be available with their former technique. The fact that the OR state is readily discernible on the electronic board and that alerts to potential problems appear as simple red markers is a very valuable improvement impossible to achieve with the traditional whiteboard. The medical staff is most concerned about patient safety and quality of services that can be improved through such simple visible outputs.

Overall, the simplicity of the implementation and communication with the MCPS is what may make the system useful to and appreciated by all the stakeholders.

\section{FUTURE WORK}

Our MCPS is still in its primitive stages. While we were able to check that such development does not require FDA approval so far, we are not yet at the stage of an application that can reach the market and pass all requirements of a standard hospital system. Our research goal and future work will be to demonstrate that MCPSs offer a great new opportunity to improve surgical patient flow management.

For example, a daily challenge is the rescheduling activity due to either cancellation or emergencies or unanticipated delays. We provide a computerized tool to help the board runner find time to add an additional case in the schedule of the day. In parallel, the smartOR knows what the current status of each surgery is. This should be enough input data to rerun the optimization OR allocation software in real time (Denton et al. 2007). In this way, one can deliver the proposition of rescheduling and leverage the awareness level of the whole operative area by creating a real link between management, leadership, and the clinical staff.

We link both technologies through a cyber-physical system (Figure 10). Wireless sensors and the probe send raw data to their controllers, which process, analyze, and filter information to save important events in the main database. This database may also be used afterward to deliver statistics to the management of the hospital. We are working on the authorization for clinical trials and integration of BoardProbe in a surgical suite connected to the smartOR. This will enable us to quantify the performance of the system and to assess the quality of the new surgical flow management.

For security reasons and patient privacy concerns, there should be a different level of access to the data. The data coming from the smartOR without information from the EMR is completely irrelevant and does not constitute an issue. However, once sent to the BoardProbe that has access to the EMR, patient privacy has to be respected. For example, there will not be any patient information on the door visualization system; information on the electrical board will be the same as the one on the whiteboard. The communication between the two systems will be encrypted and access to the global system will be possible only with credentials and identification.

We have so far followed the simplest implementation of our system. This is probably sufficient to deliver a quick proof of concept to our physicians; we suggest that only cloud-based MCPS components would offer the capability to facilitate exchange with providers, payers, and patients. Thus, we are revising our implementation to migrate to the cloud, making cyber-security an important part of the research project, and standards of cloud security will be used.

Finally, we plan to build our MCPS for OR management to be multiscale ready. We have the capacity to catch events at different scale such as the OR with the smartOR described here, but 
also at the scale of the surgical procedure itself with our smart trocar that can detect the step of the surgery and alert of any complications (Toti et al. 2014; Joerger et al. 2017b) and, also the perioperative area with Real-Time Location System. The surgical flow in an OR suite is a very complicated system that has eluded optimization for good reason. Each of these scales matters and most of the information is currently not available. We believe that MCPS, combined with the proper predictive simulation model, might be able to provide the necessary glue to integrate all of these scales into a surgery management system.

\section{ACKNOWLEDGMENT}

We would like to thank the Dunn OR crew at Houston Methodist Hospital who participated actively in this study, and Dr. V. Kallhoff, managing director of the Center for Computational Surgery team, for her numerous edits and feedback.

\section{REFERENCES}

Annette Erichsen Andersson, Ingrid Bergh, Jón Karlsson, Bengt I. Eriksson, and Kerstin Nilsson. 2012. Traffic flow in the operating room: An explorative and descriptive study on air quality during orthopedic trauma implant surgery. American fournal of Infection Control 40, 8 (2012), 750-755.

Aaron Bangor, Philip Kortum, and James Miller. 2009. Determining what individual SUS scores mean: Adding an adjective rating scale. Journal of Usability Studies 4, 3 (2009), 114-123.

Jakob E. Bardram. 2009. Activity-based computing for medical work in hospitals. ACM Transactions on Computer-Human Interaction 16, 2 (June 2009), Article 10, 36 pages.

Jakob E. Bardram and Claus Bossen. 2005. A web of coordinative artifacts: Collaborative work at a hospital ward. In Proceedings of the 2005 International ACM SIGGROUP Conference on Supporting Group Work. 168-176.

Jakob E. Bardram and A. Niels Nørskov. 2008. Context-aware patient safety system for the operating room. In Proceedings of the 10th International Conference on Ubiquitous Computing. DOI : 10.1145/1409635.1409672

Stacy Branham, Gene Golovchinsky, Scott Carter, and Jacob T. Biehl. 2010. Let's go from the whiteboard: Supporting transitions in work through whiteboard capture and reuse. In Proceedings of the SIGCHI Conference on Human Factors in Computing Systems. ACM, 75-84.

Claus Bossen and Erik Grönvall. 2015. Collaboration in-between: The care hotel and designing for flexible use. In Proceedings of the 18th ACM Conference on Computer Supported Cooperative Work \& Social Computing (CSCW'15). 1289-1301.

John Brooke. 1996. SUS-A quick and dirty usability scale. Usability Evaluation in Industry 189 (1996), 4-7.

Alfred Cuschieri. 2006. Nature of human error: Implications for surgical practice. Annals of Surgery 244, 5 (2006), 642-648.

Deborah S. Debono, David Greenfield, Joanne F. Travaglia, Janet C. Long, Deborah Black, Julie Johnson, and Jeffrey Braithwaite. 2013. Nurses' workarounds in acute healthcare settings: A scoping review. BMC Health Services Research 13, 1 (2013), 175.

William E. Deming. 1986. Out of the crisis, Massachusetts Institute of Technology. Center for Advanced Engineering Study, Cambridge, MA, 510.

Brian Denton, James Viapiano, and Andrea Vogl. 2007. Optimization of surgery sequencing and scheduling decisions under uncertainty. Health Care Management Science 10, 1 (2007), 13-24.

Richard H. Epstein, Franklin Dexter, and Elizabeth Piotrowski. 2008. Automated correction of room location errors in anesthesia information management systems. Anesthesia \& Analgesia 107, 3 (2008), 965-971.

Ayca S. Erdogan, Brian T. Denton, J. J. Cochran, L. A. Cox, P. Keskinocak, J. P. Kharoufeh, and J. C. Smith. 2011. Surgery planning and scheduling. In Wiley Encyclopedia of Operations Research and Management Science.

Geraldine Fitzpatrick and Gunnar Ellingsen. 2013. A review of 25 years of CSCW research in healthcare: Contributions, challenges and future agendas. Computer Supported Cooperative Work 22, 4-6 (August 2013), 609-665.

Armour R. Forse, J. D. Bramble, and Robert McQuillan. 2011. Team training can improve operating room performance. Surgery 150, 4 (2011), 771-778.

Marc Garbey, Guillaume Joerger, Albert Huang, Remi Salmon, Jinsu Kim, Vadim Sherman, Brian Dunkin, and Barbara Bass. 2015. An intelligent hospital operating room to improve patient health care. fournal of Computational Surgery 2 , 1 (2015), 1 .

Marc Garbey, Guillaume Joerger, Juliette Rambourg, Brian Dunkin, and Barbara Bass. 2017. Multiscale modeling of surgical flow in a large operating room suite: Understanding the mechanism of accumulation of delays in clinical practice. In Procedia Computer Science.

Francesca Guerriero and Rosita Guido. 2011. Operational research in the management of the operating theatre: A survey. Health Care Management Science 14, 1 (2011), 89-114. 
Anthony P. Hamins. 2014. Smart firefighting. In Workshop Summary Report.

Albert Y. Huang, Guillaume Joerger, Vid Fikfak, Remi Salmon, Brian J. Dunkin, Barbara L. Bass, and Marc Garbey. 2017. The smartOR: A distributed sensor network to improve operating room efficiency. Surgical Endoscopy 31, 9 (2017), 3590-3595.

Albert Y. Huang, Guillaume Joerger, Remi Salmon, Brian Dunkin, Vadim Sherman, Barbara L. Bass, and Marc Garbey. 2016. A robust and non-obtrusive automatic event tracking system for operating room management to improve patient care. Surgical Endoscopy 30, 8 (2016), 3638-3645.

Hilary Hutchinson, Wendy Mackay, Bosse Westerlund, Benjamin B. Bederson, Allison Druin, Catherine Plaisant, Michel Beaudouin-Lafon, Stéphane Conversy, Helen Evans, Heiko Hansen, Nicolas Roussel, and Björn Eiderbäck. 2003. Technology probes: Inspiring design for and with families. In Proceedings of the SIGCHI Conference on Human Factors in Computing Systems. 1724.

Guillaume Joerger, Juliette Rambourg, Marc Garbey, Stephane Conversy, and Helene Gaspard-Boulinc. 2017a. Re-engineer operating room data acquisition and transmission for improving surgical suite awareness and management. In IEEE International Conference on Biomedical and Health Informatics.

Guillaume Joerger, Albert Huang, Barbara Bass, Brian Dunkin, and Marc Garbey. 2017b. Global laparoscopy positioning system with a smart trocar. In 2017 IEEE 17th International Conference on Bioinformatics and Bioengineering (BIBE). IEEE, 359-366.

Stamatis Karnouskos. 2011. Cyber-physical systems in the smartgrid. In 2011 9th IEEE International Conference on Industrial Informatics. IEEE, 20-23.

R. Koppel and C. U. Lehmann. 2014. Implications of an emerging EHR monoculture for hospitals and healthcare systems. Journal of the American Medical Informatics Association 22, 2 (2014), 465-471.

Sophie D. Lapierre, Charlotte Batson, and Suzanne McCaskey. 1999. Improving on-time performance in health care organizations: a case study. Health Care Management Science 2, 1 (1999), 27-34.

Insup Lee, Oleg Sokolsky, Sanjian Chen, John Hatcliff, Eunkyoung Jee, BaekGyu Kim, Andrew King, Margaret MullenFortino, Soojin Park, Alexander Roederer, and Krishna Venkatasubramanian. 2012. Challenges and research directions in medical cyber-physical systems. Proceedings of the IEEE 100, 1 (2012), 75-90. Retrieved from http://dx.doi.org/10.1109/ JPROC.2011.2165270.

Tao Li, Jiannong Cao, Junbin Liang, and Junhao Zheng. 2015. Towards context-aware medical cyber-physical systems: Design methodology and a case study. Cyber-Physical Systems 1, 1 (2015), 5-23. DOI : 10.1080/23335777.2014.972686

Charles C. H. Liu, Chia-Huang Chang, Mu-Chun Su, Hsueh-Ting Chu, Sheng-Hui Hung, Jau-Min Wong, and Pa-Chun Wang. 2011. RFID-initiated workflow control to facilitate patient safety and utilization efficiency in operation theater. Computer Methods and Programs in Biomedicine 104, 3 (2011), 435-442.

Alex Macario. 2010. What does one minute of operating room time cost? Journal of Clinical Anesthesia 22, 4 (2010), $233-236$.

Alex Macario, Franklin Dexter, and Rodney D. Traub. 2001. Hospital profitability per hour of operating room time can vary among surgeons. Anesthesia \& Analgesia 93, 3 (2001), 669-675.

Ponemon Institute Research Report. 2011. Benchmark study on patient privacy and data security. fournal of Healthcare Protection Management: Publication of the International Association for Hospital Security 27, 1 (2011), 69.

Nellipudi S. R. K. Prasad and Arepalli Rajesh. 2012. RFID-based hospital real time patient management system. International Journal of Computer Trends and Technology 3, 3 (2012), 1011-1016.

Krishna Sampigethaya and Radha Poovendran. 2013. Aviation cyber-physical systems: Foundations for future aircraft and air transport. Proceedings of the IEEE 101, 8 (2013), 1834-1855.

Peter G. Scupelli, Yan Xiao, Susan R. Fussell, Sara Kiesler, and Mark D. Gross. 2010. Supporting coordination in surgical suites: Physical aspects of common information spaces. In Proceedings of the SIGCHI Conference on Human Factors in Computing Systems. 1777-1786.

Anthony Tang, Joel Lanir, Saul Greenberg, and Sidney Fels. 2009. Supporting transitions in work: Informing large display application design by understanding whiteboard use. In Proceedings of the ACM 2009 International Conference on Supporting Group Work. 149-158.

Giulia Toti, Marc Garbey, Vadim Sherman, Barbara L. Bass, and Brian J. Dunkin. 2015. A smart trocar for automatic tool recognition in laparoscopic surgery. Surgical Innovation 22, 1 (2015), 77-82.

Christoph Urban and Bernd Schmidt. 2001. PECS-agent-based modelling of human behaviour. In Emotional and IntelligentThe Tangled Knot of Social Cognition. AAAI Fall Symposium Series, North Falmouth, MA. Retrieved from http://www. aaai.org/Papers/Symposia/Fall/2001/FS-01-02/FS01-02-027.pdf.

Yan Xiao, Peter Hu, Hao Hu, Danny Ho, Franklin Dexter, Colin F. Mackenzie, F. Jacob Seagull, and Richard P. Dutton. 2005. An algorithm for processing vital sign monitoring data to remotely identify operating room occupancy in real-time. Anesthesia \& Analgesia 101, 3 (2005), 823-829.

Received August 2016; revised May 2017; accepted September 2017 